\title{
COVERING BY DISMEMBERED CONVEX DISCS
}

L. FEJES TÓTH

Let us consider a great number of convex discs not "too different" from one another about which, apart from their total area, no further data are known.

What can be said about the area of the largest square (or any other domain of given shape) which can be covered with the aid of these discs if we are allowed to cut each of them into a given number of suitable pieces?

Analogously, we can raise the problem of estimating the area of the smallest square into which the pieces of the discs can be placed without overlapping if each disc is allowed to be cut into a given number of convenient pieces.

These are; roughly expressed, the problems we intend to deal with in the present note.

1. Notations and definitions. For domains and for their area the same notation will be used.

Let $S$ be an infinite set of convex discs $d_{1}, d_{2}, \ldots$ the set of the radii of the incircles and circumcircles of which has a positive lower and a finite upper bound $r$ and $R$, respectively. We shall call such a set a normal set.

Let $s_{n}=d_{1}+\cdots+d_{n}$ be the total area of the discs $d_{1}, \cdots, d_{n}$, $t_{n}\left(\leqq s_{n}\right)$ the area of the largest domain similar to a given convex domain $t$ which can be completely covered by $d_{1}, \cdots, d_{n}$, and $T_{n}\left(\geqq s_{n}\right)$ the area of the smallest domain similar to $t$ into which the $\operatorname{discs} d_{1}, \cdots, d_{n}$ can be placed without overlapping.

Consider the positive values less than or equal to 1 ,

$$
\begin{gathered}
e=e(S)=\lim \inf t_{n} / s_{n}, \\
E=E(S)=\lim \inf s_{n} / T_{n},
\end{gathered}
$$

which can easily be shown to be independent of the choice of the domain $t$. We shall call $e$ the economy of the set $S$ with regard to covering, $E$ the economy of the set with regard to packing.

Let $S$ be a set of congruent triangles, quadrangles, or hexagons having a center of symmetry, or any other set of congruent polygons by which the plane can be tesselated. For such a set $S$ we have obviously $e(S)=E(S)=1$.

A nontrivial example is furnished by a set of equal circles, in which

Received by the editors October 12, 1949. 
case-according to researches of $R$. Kershner $[1]^{1}$ and $A$. Thue $[1,2], \quad$ respectively $-e=27^{1 / 2} / 2 \pi=0.8269 \ldots$ and $E=\pi / 12^{1 / 2}$ $=0.9069 \ldots$ holds. Various generalizations or new proofs of these equalities can be found, for example, in the papers of $\mathrm{H}$. Hadwiger [1], B. Segre-K. Mahler [1], and L. Fejes Tóth $[1,2,3]$.

Now we derive from $S$ a new set by dividing each disc $d_{i}$ into $k$ convex pieces $d_{i}^{1}, \cdots, d_{i}^{k}$. The new set $d_{1}^{1}, \cdots, d_{1}^{k}, d_{2}^{1}, \cdots, d_{2}^{k}, \cdots$ ceases generally to be a normal set but it is easy to show that it has economies $e_{k}(\geqq e)$ and $E_{k}(\geqq E)$ in the above sense independent $t^{*}$ of the domain $t$.

In what follows we shall choose $t$ to be a square.

2. Results. Let us now announce our results, which read as follows.

The members of an arbitrary normal set of convex discs can always be cut into $k(=1,2, \cdots)$ convex pieces so that the derived set of pieces has economy with regard to covering

$$
e_{k} \geqq \frac{k+1}{\pi} \sin \frac{\pi}{k+1}
$$

or so that the set of pieces has an economy with regard to packing

$$
E_{k} \geqq \frac{\pi}{2 k} \cot \frac{\pi}{2 k}
$$

In case each disc has a center of symmetry and $k$ is odd, the above inequalities can be improved as follows:

$$
\begin{gathered}
e_{k} \geqq \frac{k+2}{\pi} \sin \frac{\pi}{k+2}, \\
E_{k} \geqq \frac{\pi}{2 k+2} \cot \frac{\pi}{2 k+2} .
\end{gathered}
$$

For instance, by cutting each disc into 40 suitable pieces there always can be obtained a covering by which only one-thousandth of the area of the discs is lost $(e=0.999)$. Analogously, in order to obtain a packing by which only one-thousandth of the disposable area may remain unused $(E=0.999)$, it will always suffice to cut each disc into 29 suitable pieces.

It seems probable that dismemberments into "essentially" smaller number of pieces than 40 and 29, respectively, will generally not suffice in order to obtain economies greater than or equal to 0.999 .

\footnotetext{
${ }^{1}$ Numbers in brackets refer to the references cited at the end of the paper.
} 
More precisely, it is to be expected that (1) and (2) give exact asymptotic estimates of $1-e_{k}$ and $1-E_{k}$ for large values of $k$.

3. The case $k=1$. The case $k=1$, that is, the consideration of the original set, deserves special attention. In $\$ 4$, we shall show that the lower bound of the economies $e(S)$ and $E(S)$ of all normal sets $S$ equals the lower bound of $e(\bar{S})$ and $E(\bar{S})$ of all sets $\bar{S}$ consisting of congruent convex discs. Therefore the various known inequalities concerning the densities of lattice-like arrangements of congruent discs give lower bounds of $e$ and $E$ concerning any normal set. Let us compare the bounds obtained in this way with our results.

The inequality (1) gives only $e_{1} \geqq 2 / \pi=0.6366 \ldots$, whereas (2) becomes the trivial inequality $E_{1} \geqq 0$. On the other hand, results of I. Fáry [1] show that $e_{1}, E_{1}>2 / 3$. The exact lower bounds are unknown and their determination seems to involve considerable difficulties.

Let us now consider the case of discs having a center of symmetry.

Inequality (4) yields $E_{1} \geqq \pi / 4=0.7853 \ldots$, which can be improved -by means of researches of K. Mahler [1] and the author [4]-up to $E_{1}>3^{1 / 2} / 2=0.8660 \ldots$. The exact bound is unknown but researches of K. Reinhardt [1] and K. Mahler [2] make it probable that the best possible estimation is $E_{1} \geqq\left(9-32^{1 / 2}-\log 2\right) /\left(8^{1 / 2}-1\right)$ $=0.9024 \cdots$. It is worth noting that the constant on the right is less than the value of $E$ for a set of equal circular discs.

On the other hand, (3) gives the exact bound $e_{1} \geqq 27^{1 / 2} / 2 \pi$ $=0.8269 \cdots$, where the right side equals the value of $e$ for a set of equal circles. This inequality, which expresses a remarkable extremum property of the ellipse, was previously found by the author [5].

4. Sets of congruent discs. Let $\bar{e}$ be the lower bound of the economies $e(\bar{S})$, where $\bar{S}$ ranges over all sets of congruent convex discs. We show that for any normal set $S$ of convex discs $d_{1}, d_{2}, \cdots$ we have $e(S) \geqq \bar{e}$. The same fact holds concerning $E$.

By means of this result we have, for instance, the nontrivial fact that, for any normal set of quadrangles, $e=E=1$.

The proof rests on a well known theorem of $M$. Fréchet [1] concerning compact sets, from which it follows that the members of a set of convex domains lying in a given circle can always be divided into a finite number of classes, the members of the same class having a prescribed small "deviation" from one another. The fact that the considered set of convex domains is compact follows from Blaschke's [1] well known Auswahlsatz. 
It follows immediately that for any $\alpha<1$ there can be given a finite number $N$ of convex discs $D_{1}, \cdots, D_{N}$ and a distribution of the discs $d_{1}, d_{2}, \ldots$ into $N$ classes as well, so that if $d_{l}$ is a disc belonging, say, to the $i$ th class, then $D_{i} \geqq \alpha d_{l}$ and $D_{i}$ can be completely covered by $d_{l}$.

Let us now fix the value $\alpha$. Since a finite number of discs has no influence on the value $e(S)$, we can suppose that each class contains an infinite number of discs.

Let $n_{i}$ be the number of the discs of the $i$ th class having an index less than or equal to $n$. We shall denote the system of these discs, together with the total area of them, by $s_{n}^{i}$. Let furthermore $t_{n}^{i}$ be the largest square which can be covered by $s_{n}^{i}$, and $\bar{t}_{n}^{t}$ the largest square which can be covered by $n_{i}$ discs congruent to $D_{i}$. Since $t_{n}^{i} \geqq \bar{t}_{n}^{i}$, we have by definition of $\bar{e}$

$$
\lim \inf t_{n}^{t} / n_{i} D_{i} \geqq \lim \inf \tilde{t}_{n}^{t} / n_{i} D_{i} \geqq \bar{e}, \quad i=1, \cdots, N .
$$

Consequently there can be found an index $\nu$ so that for all $n>\nu$

$$
t_{n}^{i} \geqq \alpha n_{i} D_{i} \bar{e} \geqq \alpha^{2} \bar{e} s_{n}^{i}, \quad i=1, \cdots, N,
$$

holds.

Let us now divide the squares $t_{n}^{1}$ and $t_{n}^{2}$ by three straight line cuts in to five pieces which can be put together to make a single square. This can be effected in various ways (see, for example, Rouse BallCoxeter [1]). Then unite the arising square and $t_{n}^{3}$ in the same manner as above to a new square. By continuing this process we obtain a dissection of the squares $t_{n}^{1}, \cdots, t_{n}^{N}$ by $3(N-1)$ cuts into a certain number of parts which can be put together to form one square $t_{n}^{\prime}$. The system $L$ of the straight lines lying in $t_{n}^{\prime}$ which corresponds to the cuts has a total length less than $3(N-1)\left(2 t_{n}^{\prime}\right)^{1 / 2}$ $<3(N-1)\left(2 \pi R^{2} n\right)^{1 / 2}=O\left(n^{1 / 2}\right)$, where $R$ is the upper bound of the radii of the circumcircles of the discs.

Since by the cuts a certain number of the covering discs are injured, we do not know whether the square $t_{n}^{\prime}$ can or cannot be covered by the discs $d_{1}, \cdots, d_{n}$. But the injured discs all lie in the parallel domain of the system $L$ at distance $2 R$, which can be covered by a number $\mu_{n}=O\left(n^{1 / 2}\right)$ of circles of radius $r$, where $r$ is the lower bound of the incircle radii of the discs. Consequently $t_{n}^{\prime}$ can be covered by the discs $d_{1}, \cdots, d_{n}, d_{n+1}, \cdots, d_{n+\mu_{n}}$, where $\mu_{n}=O\left(n^{1 / 2}\right)$.

Denoting by $t_{n}$ the largest square which can be covered by the system $s_{n}$ of the $\operatorname{discs} d_{1}, \cdots, d_{n}$, we have obviously $t_{n}=O(n)$. Thus 
$\lim \inf t_{n} / s_{n}=\lim \inf t_{n+\mu_{n}} / s_{n} \geqq \lim \inf t_{n}^{\prime} / s_{n} \geqq \alpha^{2} \bar{e}$.

Since this holds for any $\alpha<1$, we have $\lim \inf t_{n} / s_{n} \geqq \bar{e}$, q.e.d.

Our statement concerning the economy $E$ can be proved analogously.

Analogous arguments show that the lower bound of the economies of the sets of pieces which arise by the most suitable dismemberments into $k$ pieces of the discs of different normal sets equals the corresponding lower bound of the economies of the sets of the pieces generated only from sets of congruent convex discs.

Therefore we restrict ourselves in what follows to sets of congruent discs.

5. An extremum property of the ellipse. We shall make use of the following known results.

If $u_{n}$ denotes the $n$-gon of the largest area inscribed in a convex domain $d, U_{n}$ the $n$-gon of the smallest area circumscribed about $d$, then

$$
\begin{array}{ll}
u_{n} \geqq d \frac{n}{2 \pi} \sin \frac{2 \pi}{n}, & n \geqq 3, \\
U_{n}<d \frac{n-2}{\pi} \tan \frac{\pi}{n-2}, & n \geqq 5 .
\end{array}
$$

Equality holds in (5) only if $d$ is an ellipse. On the other hand, the inequality $U_{n} \leqq d(n / \pi)$ tan $(\pi / n)$-which would express an extremum property of the ellipse analogous to (5)-does not hold.

The case $n=3$ of (5) was first proved by W. Blaschke [2]. His proof holds also for an arbitrary $n$, but this fact was not pointed out by him. On the other hand, Blaschke's proof of the unicity of the solution of the extremum problem in question cannot be extended immediately from $n=3$ to an arbitrary $n$.

Later on a more elementary new proof of (5) was given by E. Sas [1], which was completed by the author with a proof of the unicity valid for any number of vertices $n$.

The inequality (6) can be proved by means of a convenient modification of the proof of Sas as was shown by the author [6].

(Let us still note on this occasion that Blaschke's proof involves also the following interesting fact: In any convex body of volume $v$ there always can be inscribed an $n$-verticed polyhedron the volume of which is greater than or equal to the volume of the $n$-verticed polyhedron of maximal volume inscribed into an ellipsoid of volume $v$.) 
6. Proofs. As we have mentioned we can restrict ourselves to a set of discs congruent to a domain $d$.

Consider the polygon $p \equiv A_{1} A_{2} \cdots A_{2 k+2}$ having the largest area among the polygons of $2 k+2$ vertices inscribed in $d$. Let us divide $p-$ for example, by the cuts $A_{1} A_{4}, A_{2 k+2} A_{5}, \cdots, A_{k+2} A_{k+5}$-into $k$ convex quadrangles. We assert that the decomposition of each disc involved by the above dismemberment of $p$ will answer the purpose.

For, denoting the largest square which can be covered by the $n k$ quadrangles derived by the above dissection of $n$ polygons congruent to $p$ by $\tau_{n}$, we have lim inf $\tau_{n} / n p=1$. But $p$ satisfying the inequality $p / d \geqq((k+1) / \pi) \sin (\pi /(k+1))$, we have

$$
\lim \inf t_{n} / n d \geqq \lim \inf \tau_{n} / n d \geqq \frac{k+1}{\pi} \sin \frac{\pi}{k+1} .
$$

This completes the proof of (1).

The inequality (3) follows from the fact that the construction of Sas yields, in the case of an even $n$ and a domain $d$ which has a center of symmetry, a polygon $u_{n}$ satisfying (5) and having likewise a center of symmetry. Consequently we can inscribe in $d$ a polygon $p$ of $2 k+4$ vertices having a center of symmetry so that $p / d \geqq((k+2) / \pi)$ sin $(\pi /(k+2))$. Now we can decompose $p$ into $k-1$ quadrangles and a hexagon having a center of symmetry. But since the plane can be filled out also by such hexagons, the proof runs in the same manner as above.

The proofs of (2) and (4) run analogously, by taking into consideration instead of the inscribed polygons the circumscribed ones and using instead of (5) the inequality (6).

\section{W. BLASCHKE}

\section{REFERENCES}

1. Kreis und Kugel, Leipzig, 1916.

2. Vorlesungen über Differentialgeometrie II, Berlin, 1923, \$22, p. 49. I. FARY

1. Sur la densité des réseaux de domaines convexes, Bull. Soc. Math. France vol. 78 (1950) pp. 152-161.

L. Fejes Tóth

1. Über dichteste Kreislagerung und dilnnste Kreisilberdeckung, Comment. Math . Helv. vol. 23 (1949) pp. 342-349.

2. On the densest packing of spherical caps, Amer. Math. Monthly vol. 56 (1949) pp. 330-331.

3. Some packing and covering theorems, Acta Univ. Szeged. vol. 12A (1950) pp. 62-67.

4. On the densest packing of convex domains, Koninklijke Nederlandsche Akademie van Wetenschappen. Proceedings vol. 51 (1948) pp. 189-192.

5. Eine Bemerkung ibber die Bedeckung der Ebene durch Eibereiche mit Mittelpunkt, 
Acta Univ. Szeged. vol. 11 (1946) pp. 93-95.

6. Eine Bemerkung zur Approximation durch n-Eckringe, Compositio Math. vol. 7 (1940) pp. 474-476.

M. FRECHET

1. Les espaces abstraites, Paris, 1928.

H. HADWIGER

1. Über extremale Punktverteilungen in ebenen Gebieten, Math. Zeit. vol. 49 (1944) pp. 370-373.

R. KERSHNER

1. The number of circles covering a set, Amer. J. Math. vol. 61 (1939) pp. 665-671. K. MAHLER

1. The theorem of Minkowski-Hlawka, Duke Math. J. vol. 13 (1946) pp. 611-621.

2. On the area and the densest packing of convex domains, Koninklijke Nederlandsche Akademie van Wetenschappen. Proceedings vol. 50 (1947) pp. 108-118.

K. REINHARDT

1. Über die dichteste gitterförmige Lagerung kongruenter Bereiche in der Ebene und eine besondere Art konvexer Kurven, Abh. Math. Sem. Hamburgischen Univ. vol. 10 (1934) pp. 216-230.

W. W. Rouse Ball and H. S. M. Coxeter

1. Mathematical recreations and essays, 11 th ed., London, 1939, p. 87.

E. SAS

1. Über eine Extremumeigenschaft der Ellipsen, Compositio Math. vol. 6 (1939) pp. 468-470.

B. Segre and K. Mahler

1. On the densest packing of circles, Amer. Math. Monthly vol. 51 (1944) pp. 261270.

A. Thue

1. Om nogle geometrisk taltheoretiske Theoremer, Naturforskerm $\phi \mathrm{de}, 1892, \mathrm{pp} .352-$ 353.

2. Über die dichteste Zusammenstellung von kongruenten Kreisen in einer Ebene, Christiania Videnskabernes Selskabs Skrifter, 1910, pp. 3-9.

BUDAPEST, HuNGARY 